\title{
CONSOLIDATION OF MOTOR MEMORY
}

\author{
John W. Krakauer ${ }^{1}$ and Reza Shadmehr ${ }^{2}$ \\ 1 The Neurological Institute, Columbia University College of Physicians and Surgeons, 710 West $168^{\text {th }}$ Street, \\ New York NY 10032 \\ 2Department of Biomedical Engineering, Johns Hopkins University School of Medicine, 720 Rutland Ave, \\ 419 Traylor Building, Baltimore, MD 21205
}

\begin{abstract}
A question of great recent interest is whether motor memory consolidates in a manner analogous to declarative memories, with the formation of a memory that progresses over time from a fragile state, susceptible to interference by a lesion or a conflicting motor task, to a stabilized state, resistant to such interference. Here, we first review studies that examine the anatomical basis for motor consolidation: evidence implicates cerebellar circuitry for two types of associative motor learning, eyelid conditioning and vestibulo-ocular reflex adaptation, and primary motor cortex for skilled finger movements. We then review evidence for and against a consolidation process for adaptation of arm movements. We propose that contradictions have arisen because consolidation can be masked by inhibition of memory retrieval.
\end{abstract}

\section{Introduction}

It is clear from experience that the nervous system has the capacity to form multiple long-term (> 24 hours) motor memories, for example we apparently never forget how to swim, ride a bicycle or drive a car. However, despite the ubiquity of long-term motor memory in everyday life, experimental demonstration of how long-term motor memory is formed and retained has proven controversial. This review will focus on the specific issue of consolidation of motor memory, with consolidation defined as a set of processes whereby a long-term memory becomes more stable with the passage of time [1]. This definition implies that consolidation is proven by demonstration of a limited time-window of susceptibility of the memory to disruption by a competing memory or a focal lesion.

Motor memory differs from declarative memory in that it is demonstrated through savings in performance over several trials rather than through recall of a single item (single trial memory). Savings refers to a more rapid rate of re-learning compared to the rate of original learning. Thus a motor memory has consolidated when there is a limited time window within which savings can be disrupted. In this review, we first describe studies of simple associative motor learning for two well-characterized reflexes, where there is good evidence for consolidation and insight into its underlying neural circuitry. We then address consolidation of skilled sequential finger movements. Finally, we examine adaptation of arm movements to novel dynamics and new visuospatial mappings, two types of motor learning for which evidence for consolidation is more problematic. 


\section{Consolidation of associative motor learning}

Experimental investigation of Pavlovian eyelid conditioning (EC) and vestibular ocular reflex (VOR) adaptation in several species has provided valuable insights into the neural circuitry, specifically in the cerebellum, for savings [2] and consolidation of motor memory [3-5]. EC and VOR adaptation are similar in that they both involve associative learning in a reflex circuit [6], in which a conditioned stimulus (CS, tone or head motion) is paired with an unconditioned stimulus (US, air puff or retinal slip).

In EC, the CS is often a tone, which is followed after 100 to $1500 \mathrm{~ms}$ by a puff of air, the US. After 100-200 trials, the CS elicits precisely timed eyelid closure that precedes the US [7]. This defensive conditioned response (CR) can persist for long periods in the absence of exposure to the CS but can be extinguished by relatively few trials of the CS without the US $[8,9]$.

Extinction does not erase the motor memory, however, as relearning of the CR shows savings $[2,10]$. The degree of savings on reacquisition of the $\mathrm{CR}$ after extinction is strongly correlated with the amount of residual plasticity in the deep cerebellar nuclei. This is detected by eliciting fixed short-latency responses to the CS, after disconnection of cerebellar cortex by injection of a GABA antagonist into the cerebellar nucleus. Importantly, disconnection of the cerebellar cortex from the interpositus prevents extinction of EC [11]. This suggests a "cascade" model of adaptation where the CS and the error signals initially produce rapid plasticity in the cerebellar cortex, resulting in a change in the discharge of the Purkinje cells. Subsequently, the altered output of the Purkinje cells in combination with the CS signals produce gradual plasticity in the interpositus neurons $[2,3,12]$. Therefore, the learned behavior in EC may be due to a system that adapts quickly (cerebellar cortex) and then subsequently produces plasticity in another system (the cerebellar nuclei).

Passage of time affects the way that the cerebellar cortex contributes to retention of EC. Animals that receive muscimol infusion to the cerebellar cortex at 5 minutes or 45 minutes after acquisition of the CR show no savings of EC [4,5]. In contrast, control animals and animals that receive muscimol after 90 minutes resume in a second acquisition phase where they left off in the first acquisition phase. This suggests that soon after completion of training, adapted behavior strongly depends on the plasticity in the cerebellar cortex. However, passage of time reduces or eliminates this dependence.

Work on retention of VOR adaptation in also compatible with the cascade model. Lesion of the flocculus prevents adaptation of the horizontal VOR [13] and reversible inactivation of the flocculus abolishes retention of a VOR gain acquired over 1-3 hours [14-16]. In contrast, flocculus inactivation fails to abolish a VOR gain acquired over days [16-19]. It seems plausible that learned behavior initially depends on the modified synapses in the cerebellar cortex, but that with time the location of the memory shifts. Eventually, certain adapted behaviors may become independent of the cerebellum entirely. For example, in fear conditioning, the ability of a rat to recall the importance of an acoustic cue depends on the integrity of the interpositus for about 96 hours after initial training [20]. After that interval, disruption of the interpositus has no effect on recall. Is there evidence for this time dependent role of the cerebellum in humans? A recent study examined cerebellar patients with cerebellar cortex lesions in two different kinds of EC tasks: one task that was novel (associating tone with an eye blink), and one task that the patients had learned before their cerebellar cortex injury (a kinesthetic threat eye blink response, called KTER) [21]. The patients could not learn the new conditioning task, which confirmed the idea that acquisition of the new eye blink task depended on cerebellar cortex. Surprisingly, they maintained the response for the old task. This suggested that in humans, the cerebellum was required for acquisition of a new conditioned response but not for its long-term retention. Overall, these results suggest that simple motor memories undergo changes in their anatomical storage site as they stabilize, with progression over time from 
cerebellar cortex to cerebellar nuclei, and then perhaps to locations outside the cerebellum $[21,22]$.

There are reasons to suppose that the cerebellar mechanisms elucidated for consolidation of EC and VOR adaptation are relevant to motor consolidation in general. First, the regularity of synaptic organization throughout the cerebellum suggests that it performs a similar computation for many types of motor learning. Second, current evidence suggests that both saccadic and reach adaptation, voluntary movements that are not simple reflexs, are critically dependent on the cerebellum [23-26]. For example, damage to the cerebellum but not damage to the basal ganglia can profoundly impair adaptation of reaching when novel forces act on the hand [27]. Third, patient studies [24-27], animal data [28], functional imaging studies [29-32], and recent computational models [33-35] suggest a role for the cerebellum in storage of internal models for reaching movements.

\section{Consolidation of skilled finger movements}

Repetitive $1 \mathrm{~Hz}$ transcranial magnetic stimulation (rTMS) has been used to investigate the role of primary motor cortex (M1) in the retention of a simple finger opposition task [36]. Subjects performed a metronome-paced $(0.5 \mathrm{~Hz})$ ballistic pinch between the index finger and thumb of the non-dominant left hand. Performance measure was acceleration of the pinching movement, which was assessed across two practice sessions. Control subjects showed increases in peak pinch acceleration within a first practice session and subsequent savings in a second practice session performed 15 minutes later. Savings in the second session was eliminated if subjects received rTMS for the 15 minutes between the two practice sessions. The rTMS was applied over an area of right M1 selected by its capacity to elicit MEPs in the left flexor pollicis brevis, an area shown in previous studies to be associated with practice-induced changes in M1 excitability [37]. In contrast, when the 15 minutes of rTMS over M1 was given six hours after the first practice session, it had little or no effect on savings. The authors proposed that while acquisition of this skill depended on M1, its eventual retention depended on a different neural system. This is consistent with a recent observation that immediately after training in this task, it was much more difficult to produce LTP-like plasticity in human M1 than at 6 hours after completion of training [38]. Another group subsequently confirmed this result [39].

Further evidence of a consolidation window for skill learning was obtained from a study in which subjects performed a finger-tapping task in a four-digit sequence [40]. Performance was measured in two ways: speed (number of complete sequences achieved) and accuracy (number of errors made compared to the number of correct sequences). Subjects showed savings for the same sequence 24 hours later. However, if subjects learned a second sequence immediately after the first, then there was no savings (of the first sequence) at 24 hours. In contrast, subjects showed savings for both sequences if the second sequence was learned 6 hours after the first. These studies indicate that retention of certain forms of skills in humans may be initially susceptible to disruption but this susceptibility may be reduced with time. Although all these studies use a finger task, there is an important distinction to be made between studies that emphasize simple tapping or pinching skills, assessed by measuring acceleration and force, versus those studies that show an increase in accuracy and speed as a whole sequence is learned and anticipated (see Box 1). It is possible or even likely that consolidation of these two aspects of motor learning will have different anatomical substrates.

\section{Consolidation of internal models for reaching}

In the last 20 years, a computational framework has been developed to characterize behavioral and neural basis of planning and execution of reaching (for an extended treatment see [41]). It appears that the CNS plans reaching movements in extrinsic space [42-44], with target and hand location initially coded as vectors with respect to fixation that are then subtracted to 
produce an intended movement vector in a hand-centered coordinate system. The transformation of this vector into motor commands depends on maps or internal models. The computation that allows the CNS to predict the sensory consequences of motor commands (in both visual and proprioceptive coordinates) is termed a "forward model". For example, when one tries to reach while looking at the hand through a mirror, one observes a mismatch between the visually observed consequences of the motor command and its proprioceptive feedback. This error can produce adaptation in a forward model of the arm. The term "inverse model" labels the computation that allows the CNS to plan motor commands in order to achieve a goal defined in sensory coordinates. For example, lifting up an apparently a cup full of liquid relies on an inverse model that predicts the motor commands necessary to accelerate the cup upwards. Finding out that the cup was actually empty results in an error that modifies the internal model associated with that action. Motor learning likely involves adaptation of both kinds of maps [45], although in principle it is difficult to tease apart contribution of forward and inverse models to adapted behavior [46]. Here, we will use the term internal models to refer to both kinds of computations.

Many studies have shown that after an internal model is learned, it can be recalled at a later date. This is apparent as "savings": relearning is more rapid and complete than original learning. The first experiment to show consolidation of motor learning examined the formation of two competing inverse models using a well-characterized force field adaptation paradigm. Subjects who adapted to a clockwise force field (B1) during reaching showed savings when they were re-exposed to the same field after an interval of hours or days. However, savings on relearning was prevented if after learning the first field, subjects adapted to a counter-clockwise field (B2). Crucially, B2 no longer interfered with savings for B1 if the time interval between training with the two force fields was greater than about 4-6 hours $[47,48]$. These results demonstrated for the first time that motor learning showed a retrograde gradient of susceptibility to an interfering task consistent with a consolidation process. Interestingly, the ability to learn B2 became progressively better as the interval from learning B1 lengthened. Indeed, there was no significant difference between learning of B2 six hours after B1 and initial learning of B1. The time course of this improvement was attributable to fading of after-effects from B1. The similarity in the time courses of susceptibility of B2 to anterograde interference by $\mathrm{B} 1$ and susceptibility of B1 to retrograde interference by B2 suggested a possible obligatory relationship between anterograde and retrograde effects. This was addressed with an experiment in which subjects were trained with $\mathrm{B} 1$ on day 1 and then returned the next day to train again on B1 followed by B2. The degree of anterograde interference of B1 on B2 was not significantly different from the initial experiment. However, when B1 was re-tested on day 3, retrograde interference was not seen. This result is supportive but not conclusive evidence for the notion that retrograde and anterograde interference are independent non-reciprocal processes during learning of dynamics. It is not fully conclusive because if learning on day 3 had been compared to a control group who learned B1 over three days without B2 on day 2, then an interference effect might have been apparent.

Since the publication of the aforementioned study and its initial evidence for consolidation, attempts to replicate the result [49] or reproduce it [49-51] have, with one exception [52], failed. Up until very recently, visuomotor adaptation experiments have consistently shown a flat gradient of interference: task B prevented savings in task A regardless of the interval between $\mathrm{A}$ and $\mathrm{B}$. The first experiment to show a flat gradient employed a joystick-tracking task, in which the screen cursor was either inverted up-down or left-right [50]. Subjects showed savings when they repeated either task but the tasks interfered with each other even with a one-month interval between them. Similarly, interference between opposite lateral-displacing prisms, used in a ball-throwing task, persisted with intervals of 5 minutes, 1 hour, 5 hours and 24 hours [51]. If anything, interference increased as the interval increased; the opposite of what would be expected for a consolidation process. The presence of a flat gradient has led investigators 
to conclude that consolidation may not occur for visuomotor learning [49,53]. However, this conclusion is likely to be incorrect. As stated by Dudai [1], "Monotonous effectiveness of a blocking agent over time suggests that this agent impairs maintenance, retrieval or expression of memory, not its consolidation."

A recent study addressed this issue by hypothesizing that anterograde effects prevented retrieval and thus masked consolidation [52]. The prediction was that "washout" of anterograde effects by returning subjects to baseline performance would unmask a consolidation process. The motor adaptation task was a $30^{\circ}$ rotation on the usual relationship between cursor and hand movements during reaching --- a visuomotor perturbation that showed robust savings and interference [54,55]. In the first experiment, a $30^{\circ}$ rotation that was followed by a $30^{\circ}$ counterrotation prevented savings even when the two training intervals were separated by 24 hours. This result was consistent with the flat gradient of susceptibility shown in previous studies [49-51]. In the second experiment, however, introduction of baseline blocks (washout) between rotations led to interference by a counter-rotation at 5 minutes but not at 24 hours, consistent with a consolidation process. In a third experiment, when the amount of rotation training was doubled, the counter-rotation did not interfere even at 5 minutes. Thus consolidation occurred for visuomotor adaptation both through a graded effect of time interval and with increased initial training. The key factor appeared to be the washout trials. In the absence of washout, the counter-rotation prevented retrieval of the internal model for the initial rotation. A similar inhibition of retrieval effect is described in the declarative memory literature for pairedassociates word learning [56].

A final point about the washout experiment is that it provides evidence that visuomotor adaptation tasks can undergo another form of consolidation called off-line learning. Off-line learning represents improvements in motor skill without practice, i.e. between training sessions. Although this review is about consolidation as memory stabilization rather than as off-line learning, the issue is raised here for two reasons. First, to make readers aware that there are two distinct definitions of motor consolidation in the literature, and second, as a response to a recent statement that off-line improvement has not been convincingly demonstrated for visuomotor, or kinematic, adaptation tasks [53]. The reason for this statement is that better performance at re-learning cannot be taken as evidence for off-line improvement because the improvement might simply be the result of practice, i.e. resuming on the learning curve from the point reached on the previous day. However, in the washout study described above, subjects started at the same point on the curve in the re-learning session as they did at initial learning but still learned at a faster rate. This is convincing evidence for off-line learning. Thus visuomotor adaptation tasks can undergo both types of consolidation.

Experiments have not yet been done that directly address anatomical sites of consolidation for reach adaptation. However, an important clue with regard to storage and retention of internal models has been provided by single-unit recording experiments in M1 [57]. Activity in M1 neurons was averaged from just before movement onset to movement end while monkeys adapted and then de-adapted through washout in force fields. One group of neurons rotated their preferred direction (PD) in the direction of the field during adaptation and then rotated back during washout. This is similar to the behavior of muscles [58] and may merely indicate that motor commands are changing in response to the movement errors. However, two other groups of neurons showed a much more interesting response: one group showed a change in PD during field training and maintained this change despite a long stretch of washout trials. Another group did not change its PD during field training but rotated in a direction opposite to the field during washout. Therefore, if one measures the change in motor output as the sum of changes in PD, then during washout this sum was zero despite the fact the individual components were not at baseline. It can be conjectured that the presence of a population of 
neurons that do not return to baseline might form the basis for savings because they can be recruited when subjects return to the force-field condition.

\section{Consolidation of internal models in bimanual skills}

In bimanual movements, one hand often serves a supportive role by holding an object that is manipulated by the other hand. For example, when one hand holds a PDA (personal digital assistant device) while the other hand writes on it, the supporting hand shows compensatory adjustments to counteract the anticipated forces arising from the writing hand. A similar situation occurs when one hand holds a book while the other hand lifts it: the postural hand reduces the upward forces precisely at the moment when the lift starts. In this way, the postural hand shows little displacement as the object is lifted. In contrast, if another person were to lift the object, the postural hand would show large upward displacements $[59,60]$.

These types of adjustments demonstrate that when we perform an action with one arm, the brain anticipates its consequences on other body parts (an example of a forward model). For lifting an object, the skill is acquired in late childhood [61]. A recent study examined acquisition and retention of this skill in cerebellar patients [22]. They used a robot to simulate the load held by the postural hand. Surprisingly, cerebellar patients showed normal anticipatory adjustments by the postural hand as the object was lifted by the other hand. Long-term retention of the skill appeared intact in these patients. To measure acquisition of a new response, the study [22] considered a novel condition. They introduced catch trials where the normal behavior of the load was unexpectedly altered. In healthy individuals, the resulting error in the catch trial produced a robust change in the behavior of the postural hand in the subsequent trial. This trial-to-trial compensation was absent in the cerebellar patients. The results challenge the idea that the cerebellum may be the site of long-term storage of internal models that compensate for the consequence of our own actions. Rather, the results suggest that while the cerebellum is crucial for acquisition of these internal models, long-term retention may be independent of this structure.

\section{Conclusions}

There is evidence for motor consolidation in a number of experimental systems: the behavioral correlates of the memory suggest that under certain conditions, the neural substrate becomes resistant to disruption within hours or days. In certain tasks, the cerebellar cortex appears to play a crucial role in the initial storage of the memory but with time, the memory may be represented elsewhere. Because motor learning is made up of a number of distinct processes (planning of sequences of action, adaptation of internal models, etc.), they may consolidate with different time courses and at different anatomical locations. There is a precedent for this with declarative memory: semantic memories become hippocampus-independent whereas episodic memories do not [62].

Important challenges remain. First, computational models of motor control have focused mainly on the adaptation process, with little or no attempt to consider questions of timedependent memory processes. Key experiments that consistently show savings after washout are now largely unexplained [52,63]. Second, motor learning is about associating knowledge to specific contextual cues. Yet, attempts to learn cue-dependent internal models in the laboratory have suffered from large interference between opposing motor tasks with explicit contextual cues. A recent report shows that passage of time may be a key element that was missing in the earlier works [64]. It is also possible that there are as of yet unidentified implicit contextual cues that facilitate or inhibit recall of consolidated memories. Identification of those cues that prevent retrieval of consolidated memories and those that allow switching between 
consolidated memories are critical to understanding how animals are capable of forming longterm, context addressable motor memories.

\section{Box 1}

\section{The difference between skill learning and sequence learning}

It is useful to distinguish between skill and sequence learning as these two types of learning are often confused in the literature. In a recent review on consolidation of "procedural memory", it was stated "..the evidence for sequence learning tasks requiring stabilization is mixed" [53]. This statement is based on the finger tapping study by Walker et al. [40] (mentioned in the section on skilled finger movements) and a recent serial reaction time (SRT) study, which found no evidence for consolidation of sequence learning [51]. However, the statement is debatable for two reasons. First, it compares skill learning with sequence learning. Second, the SRT study of consolidation is open to question. We will briefly deal with each of these issues in turn. Finger tasks in which the sequence is short are best considered skill tasks because the performance measures of interest relate to speed of execution of the sequence not the rate of acquisition of the sequence order per se. In contrast, finger tasks in which the sequence is long and embedded (e.g a 12 element sequence with only four buttons) are based on the much-studied SRT and the measure of interest, the reaction time (RT), captures learning of the embedded sequence order itself [65]. These two aspects to sequence learning are almost certainly cognitively distinct as one is effector-specific and the other is effector-independent $[66,67]$. The inter-play of these two forms of skill learning is apparent in the SRT study that purported to show no evidence of consolidation for sequence learning. All subjects learned sequence A on day 1 and then returned 48 hours later in session 2 to be re-tested on sequence A. Unlike the control group, four other groups learned a second interfering sequence B, either 5 minutes, one hour, five hours or 24 hours after sequence A. The learning measure was the difference between the $\mathrm{RT}$ at the end of sequence learning and the RT for a random sequence. Savings was quantified by subtraction of the learning measure for sequence $\mathrm{A}$ from the learning measure for re-test of sequence $\mathrm{A}$. This measure was positive only for the control group and negative for the other four groups. Unfortunately, this apparent persistence of interference could be an artifact caused by increased skill in the random sequence, attributable perhaps to more practice overall because of additional training with sequence $\mathrm{B}$. This is suggested by the fact that for the four interference groups, the RT for a random sequence at the beginning of session 2 was actually lower than the RT for sequence A at the end of session 1 . This indicates that savings for non-specific skill component of the task led to a greater reduction in RT than learning of the sequence order itself. The presence of an interference effect may therefore have been due to the choice of learning measure, which could be affected equally by savings in non-specific skill learning or by interference with the specific sequence. The authors would have a stronger claim if they had shown that subjects, with extra training on sequence A, could lower their RT below that shown for the four interference groups i.e. show the absence of an RT floor effect for their particular task. Without showing this, the results remain ambiguous.

Box 2

\section{Outstanding issues and future directions}

i. A recent study [49] was unable to find evidence for consolidation of force field learning with or without a washout paradigm. This result reinforces the fact that anterograde interference effects, which lead to flat gradients, are a very robust phenomenon in motor learning and may not always be effectively removed by 
washout blocks. Further work is needed to characterize the factors that influence retrieval of motor memories.

ii. Experiments on consolidation of motor memories suggest a process that takes place over hours, compatible with synaptic consolidation. As of yet, there is no evidence for a process that takes places over weeks or months, as is seen with declarative memory. Further investigation is required to determine whether this represents a fundamental distinction between these two types of memory.

iii. In addition to the passage of time and amount of initial learning, it has been suggested that sleep plays a role in consolidation of motor memories. However, most studies to date have focused on finger tapping skill and consolidation has been defined as off-line learning $[68,69]$ rather than resistance to interference. The importance of distinguishing between these two definitions of consolidation is suggested by dissociation in their dependency on sleep. For example, in one study it was shown that passage of time is sufficient to render tapping skill for a particular sequence resistant to interference by another sequence but sleep is required for offline learning [40]. In another study, this time of force-field learning, no effect of sleep deprivation on performance improvements was observed [70], suggesting that the necessity of sleep even for off-line learning may vary for different types of motor learning. As of this writing, there is no evidence that sleep plays a special role in motor memory stabilization i.e. consolidation as defined in this review.

iv. Numerous functional imaging studies have shown transitions in brain areas activated over short (minutes, hours) [29,71-73] and long (days, weeks) [74,75] time courses of motor learning. However, changes in the location of activation over time are not itself indicative of consolidation as it may represent multiple processes, for example change in performance with practice. Future studies will need to correlate behavioral measures of savings and interference with activation changes in order to identify brain regions associated with motor consolidation. Functional imaging studies will need to be corroborated by virtual lesions using TMS in humans or real lesions in animal models.

\section{Acknowledgements}

This work was supported by National Institute of Neurological Disorders and Stroke Grants K23-NS02138 (JWK) and R01-NS037422 (RS). We thank Javier Medina and Michael Mauk for insightful discussions. Thanks to Toni Pearson for critical comments on the manuscript.

\section{References}

1. Dudai Y. The neurobiology of consolidations, or, how stable is the engram? Annu Rev Psychol 2004;55:51-86. [PubMed: 14744210]

2. Medina JF, Garcia KS, Mauk MD. A mechanism for savings in the cerebellum. J Neurosci 2001;21 (11):4081-9. [PubMed: 11356896]

3. Ohyama T, Mauk M. Latent acquisition of timed responses in cerebellar cortex. J Neurosci 2001;21 (2):682-90. [PubMed: 11160447]

4. Attwell PJ, Cooke SF, Yeo CH. Cerebellar function in consolidation of a motor memory. Neuron 2002;34(6):1011-20. [PubMed: 12086647]

5. Cooke SF, Attwell PJ, Yeo CH. Temporal properties of cerebellar-dependent memory consolidation. J Neurosci 2004;24(12):2934-41. [PubMed: 15044532]

6. Mauk MD. Roles of cerebellar cortex and nuclei in motor learning: contradictions or clues? Neuron 1997;18(3):343-6. [PubMed: 9115728] 
7. Christian KM, Thompson RF. Neural substrates of eyeblink conditioning: acquisition and retention. Learn Mem 2003;10(6):427-55. [PubMed: 14657256]

8. Schneiderman N, Fuentes I, Gormezano I. Acquisition and extinction of the classically conditioned eyelid response in the albino rabbit. Science 1962;136:650-652. [PubMed: 13908977]

9. Robleto K, Poulos AM, Thompson RF. Brain mechanisms of extinction of the classically conditioned eyeblink response. Learn Mem 2004;11(5):517-24. [PubMed: 15466302]

10. Napier RM, Macrae M, Kehoe EJ. Rapid reaquisition in conditioning of the rabbit's nictitating membrane response. J Exp Psychol Anim Behav Process 1992;18(2):182-92. [PubMed: 1583447]

11. Medina JF, Nores WL, Mauk MD. Inhibition of climbing fibres is a signal for the extinction of conditioned eyelid responses. Nature 2002;416(6878):330-3. [PubMed: 11907580]

12. Medina JF, et al. Parallels between cerebellum- and amygdala-dependent conditioning. Nat Rev Neurosci 2002;3(2):122-31. [PubMed: 11836520]

13. Nagao S. Effects of vestibulocerebellar lesions upon dynamic characteristics and adaptation of vestibulo-ocular and optokinetic responses in pigmented rabbits. Experimental Brain Research 1983;53:36-46.

14. McElligott JG, Beeton P, Polk J. Effect of cerebellar inactivation by lidocaine microdialysis on the vestibuloocular reflex in goldfish. J Neurophysiol 1998;79(3):1286-94. [PubMed: 9497410]

15. Nagao S, Kitazawa H. Effects of reversible shutdown of the monkey flocculus on the retention of adaptation of the horizontal vestibulo-ocular reflex. Neuroscience 2003;118(2):563-70. [PubMed: 12699790]

16. Kassardjian CD, et al. The site of a motor memory shifts with consolidation. J Neurosci 2005;25(35): 7979-85. [PubMed: 16135754]

17. Luebke AE, Robinson DA. Gain changes of the cat's vestibulo-ocular reflex after flocculus deactivation. Experimental Brain Research 1994;98(3):379-390.

18. Partsalis AM, Zhang Y, Highstein SM. Dorsal Y group in the squirrel monkey. II. Contribution of the cerebellar flocculus to neuronal responses in normal and adapted animals. J Neurophysiol 1995;73 (2):632-50. [PubMed: 7760123]

19. Broussard DM, Kassardjian CD. Learning in a simple motor system. Learn Mem 2004;11(2):12736. [PubMed: 15054127]

20. Sacchetti B, et al. Cerebellar role in fear-conditioning consolidation. Proc Natl Acad Sci U S A 2002;99(12):8406-11. [PubMed: 12034877]

21. Bracha V, et al. The human cerebellum and associative learning: dissociation between the acquisition, retention and extinction of conditioned eyeblinks. Brain Res 2000;860(12):87-94. [PubMed: 10727626]

22. Diedrichsen J, et al. Cerebellar involvement in anticipating the consequences of self-produced actions during bimanual movements. J Neurophysiol 2005;93(2):801-12. [PubMed: 15356182]

23. Hopp JJ, Fuchs AF. The characteristics and neuronal substrate of saccadic eye movement plasticity. Prog Neurobiol 2004;72(1):27-53. [PubMed: 15019175]

24. Weiner MJ, Hallett M, Funkenstein HH. Adaptation to lateral displacement of vision in patients with lesions of the central nervous system. Neurology 1983;33(6):766-72. [PubMed: 6682520]

25. Martin TA, et al. Throwing while looking through prisms. I. Focal olivocerebellar lesions impair adaptation. Brain 1996;119(Pt 4):1183-98. [PubMed: 8813282]

26. Maschke M, et al. Hereditary cerebellar ataxia progressively impairs force adaptation during goaldirected arm movements. J Neurophysiol 2004;91(1):230-8. [PubMed: 13679403]

27. Smith MA, Shadmehr R. Intact ability to learn internal models of arm dynamics in Huntington's disease but not cerebellar degeneration. J Neurophysiol 2005;93(5):2809-21. [PubMed: 15625094]

28. Lu X, Hikosaka O, Miyachi S. Role of monkey cerebellar nuclei in skill for sequential movement. J Neurophysiol 1998;79(5):2245-54. [PubMed: 9582201]

29. Shadmehr R, Holcomb HH. Neural correlates of motor memory consolidation. Science 1997;277 (5327):821-5. [PubMed: 9242612]

30. Imamizu $\mathrm{H}$, et al. Human cerebellar activity reflecting an acquired internal model of a new tool. Nature 2000;403(6766):192-5. [PubMed: 10646603] 
31. Nezafat R, Shadmehr R, Holcomb HH. Long-term adaptation to dynamics of reaching movements: a PET study. Exp Brain Res 2001;140(1):66-76. [PubMed: 11500799]

32. Imamizu $\mathrm{H}$, et al. Modular organization of internal models of tools in the human cerebellum. Proc Natl Acad Sci U S A 2003;100(9):5461-6. [PubMed: 12704240]

33. Doya K. What are the computations of the cerebellum, the basal ganglia and the cerebral cortex? Neural Netw 1999;12(78):961-974. [PubMed: 12662639]

34. Wolpert DM, Miall C, Kawato M. Internal models in the cerebellum. Trends Cogn Sci 1998;2(9): 338-347.

35. Wolpert DM, Kawato M. Multiple paired forward and inverse models for motor control. Neural Netw 1998;11(78):1317-1329. [PubMed: 12662752]

36. Muellbacher W, et al. Early consolidation in human primary motor cortex. Nature 2002;415(6872): 640-4. [PubMed: 11807497]

37. Classen J, et al. Rapid plasticity of human cortical movement representation induced by practice. J Neurophysiol 1998;79(2):1117-23. [PubMed: 9463469]

38. Stefan K, et al. Temporary Occlusion of Associative Motor Cortical Plasticity by Prior Dynamic Motor Training. Cereb Cortex. 2005

39. Baraduc $\mathrm{P}$, et al. Consolidation of dynamic motor learning is not disrupted by rTMS of primary motor cortex. Curr Biol 2004;14(3):252-6. [PubMed: 14761660]

40. Walker MP, et al. Dissociable stages of human memory consolidation and reconsolidation. Nature 2003;425(6958):616-20. [PubMed: 14534587]

41. Shadmehr, R.; Wise, SP. The computational neurobiology of reaching and pointing: a foundation for motor learning. In: Sejnowski, TJ.; Poggio, TA., editors. Computational Neuroscience. The MIT press; Cambridge, Massachusetts: 2005.

42. Gordon J, Ghilardi MF, Ghez C. Accuracy of planar reaching movements. I. Independence of direction and extent variability. Exp Brain Res 1994;99(1):97-111. [PubMed: 7925800]

43. Vindras P, Viviani P. Frames of reference and control parameters in visuomanual pointing. Journal of experimental psychology 1998;24:1-23.

44. Krakauer JW, et al. Learning of visuomotor transformations for vectorial planning of reaching trajectories. J Neurosci 2000;20(23):8916-24. [PubMed: 11102502]

45. Bhushan N, Shadmehr R. Computational nature of human adaptive control during learning of reaching movements in force fields. Biol Cybern 1999;81(1):39-60. [PubMed: 10434390]

46. Mehta B, Schaal S. Forward models in visuomotor control. J Neurophysiol 2002;88(2):942-53. [PubMed: 12163543]

47. Brashers-Krug T, Shadmehr R, Bizzi E. Consolidation in human motor memory. Nature 1996;382:252-255. [PubMed: 8717039]

48. Shadmehr R, Brashers-Krug T. Functional stages in the formation of human long-term motor memory. Journal of Neuroscience 1997;17(1):409-419. [PubMed: 8987766]

49. Caithness G, et al. Failure to consolidate the consolidation theory of learning for sensorimotor adaptation tasks. J Neurosci 2004;24(40):8662-71. [PubMed: 15470131]

50. Bock O, Schneider S, Bloomberg J. Conditions for interference versus facilitation during sequential sensorimotor adaptation. Exp Brain Res 2001;138(3):359-65. [PubMed: 11460774]

51. Goedert KM, Willingham DB. Patterns of interference in sequence learning and prism adaptation inconsistent with the consolidation hypothesis. Learn Mem 2002;9(5):279-92. [PubMed: 12359837]

52. Krakauer JW, Ghez C, Ghilardi MF. Adaptation to visuomotor transformations: consolidation, interference, and forgetting. J Neurosci 2005;25(2):473-8. [PubMed: 15647491]

53. Robertson EM, Pascual-Leone A, Miall RC. Current concepts in procedural consolidation. Nat Rev Neurosci 2004;5(7):576-82. [PubMed: 15208699]

54. Krakauer JW, Ghilardi MF, Ghez C. Independent learning of internal models for kinematic and dynamic control of reaching. Nat Neurosci 1999;2(11):1026-31. [PubMed: 10526344]

55. Wigmore V, Tong C, Flanagan JR. Visuomotor rotations of varying size and direction compete for a single internal model in motor working memory. J Exp Psychol Hum Percept Perform 2002;28(2): 447-57. [PubMed: 11999865] 
56. Wixted JT. The psychology and neuroscience of forgetting. Annu Rev Psychol 2004;55:235-69. [PubMed: 14744216]

57. Li CS, Padoa-Schioppa C, Bizzi E. Neuronal correlates of motor performance and motor learning in the primary motor cortex of monkeys adapting to an external force field. Neuron 2001;30(2):593607. [PubMed: 11395017]

58. Thoroughman KA, Shadmehr R. Electromyographic correlates of learning an internal model of reaching movements. J Neurosci 1999;19(19):8573-88. [PubMed: 10493757]

59. Massion J, et al. Acquisition of anticipatory postural adjustments in a bimanual load-lifting task: normal and pathological aspects. Exp Brain Res 1999;128(12):229-35. [PubMed: 10473765]

60. Diedrichsen J, et al. Anticipatory adjustments in the unloading task: is an efference copy necessary for learning? Exp Brain Res 2003;148(2):272-6. [PubMed: 12520418]

61. Schmitz C, Assaiante C. Developmental sequence in the acquisition of anticipation during a new coordination in a bimanual load-lifting task in children. Neurosci Lett 2002;330(3):215-8. [PubMed: 12270631]

62. Nadel L, Moscovitch M. Memory consolidation, retrograde amnesia and the hippocampal complex. Curr Opin Neurobiol 1997;7(2):217-27. [PubMed: 9142752]

63. Kojima Y, Iwamoto Y, Yoshida K. Memory of learning facilitates saccadic adaptation in the monkey. J Neurosci 2004;24(34):7531-9. [PubMed: 15329400]

64. Shelhamer M, Aboukhalil A, Clendaniel R. Context-specific adaptation of saccade gain is enhanced with rest intervals between changes in context state. Ann N Y Acad Sci 2005;1039:166-75. [PubMed: 15826971]

65. Nissen M, Bullemer P. Attentional requirements of learning: Evidence from performance measures. Cognitive Psychology 1987;19:1-32.

66. Hikosaka $\mathrm{O}$, et al. Parallel neural networks for learning sequential procedures. Trends Neurosci 1999;22(10):464-71. [PubMed: 10481194]

67. Bapi RS, Doya K, Harner AM. Evidence for effector independent and dependent representations and their differential time course of acquisition during motor sequence learning. Exp Brain Res 2000;132 (2):149-62. [PubMed: 10853941]

68. Fischer S, et al. Sleep forms memory for finger skills. Proc Natl Acad Sci U S A 2002;99(18):1198791. [PubMed: 12193650]

69. Walker MP, et al. Practice with sleep makes perfect: sleep-dependent motor skill learning. Neuron 2002;35(1):205-11. [PubMed: 12123620]

70. Donchin O, et al. Mechanisms influencing acquisition and recall of motor memories. J Neurophysiol 2002;88(4):2114-23. [PubMed: 12364533]

71. Toni I, et al. The time course of changes during motor sequence learning: a whole-brain fMRI study. Neuroimage 1998;8(1):50-61. [PubMed: 9698575]

72. Petersen SE, et al. The effects of practice on the functional anatomy of task performance. Proc Natl Acad Sci U S A 1998;95(3):853-60. [PubMed: 9448251]

73. Krakauer JW, et al. Differential cortical and subcortical activations in learning rotations and gains for reaching: a PET study. J Neurophysiol 2004;91(2):924-33. [PubMed: 14523069]

74. Karni A, et al. The acquisition of skilled motor performance: fast and slow experience-driven changes in primary motor cortex. Proc Natl Acad Sci U S A 1998;95(3):861-8. [PubMed: 9448252]

75. Doyon J, et al. Experience-dependent changes in cerebellar contributions to motor sequence learning. Proc Natl Acad Sci U S A 2002;99(2):1017-22. [PubMed: 11805340] 